\title{
An exact functional Radon-Nikodym theorem for Daniell integrals
}

\author{
by \\ E. De Amo (Almería), I. Chitescu (Bucureşti) \\ and M. Díaz CARrillo (Granada)
}

\begin{abstract}
Given two positive Daniell integrals $I$ and $J$, with $J$ absolutely continuous with respect to $I$, we find sufficient conditions in order to obtain an exact Radon-Nikodym derivative $f$ of $J$ with respect to $I$. The procedure of obtaining $f$ is constructive.

1. Introduction. In this paper we consider two positive Daniell integrals $I$ and $J$ on a lattice of functions $B$ which is also a unitary algebra, $J$ being absolutely continuous with respect to $I$. We give sufficient conditions to obtain, in a constructive manner, an "exact" Radon-Nikodym derivative $f$ of $J$ with respect to $I$, i.e., to have $J(u)=I(f u)$ for every $u$ in $B$. Generally, the derivative thus obtained must be in a larger space than $B$, so the relation $J(u)=I(f u)$ actually holds for the canonical extensions of $I$ and $J$.

We recognize the strong influence of [6] and [8].
\end{abstract}

2. Main result. We shall consider a nonempty set $X$ (the total space) and a vector lattice $B$ of functions $f: X \rightarrow \mathbb{R}$ (with pointwise operations and order). We shall also assume that $B$ is an algebra with unit $1 \in B$. We denote by $+B$ the positive elements in $B$.

In what follows, $I: B \rightarrow \mathbb{R}$ will be a positive Daniell integral (i.e., $I$ is linear, positive and $I\left(f_{n}\right) \searrow 0$ whenever the decreasing sequence $\left(f_{n}\right)$ in $B$ is such that $f_{n} \searrow 0$ pointwise). For Daniell integrals, see [9] and [10], and for general measure theory, see [5], [7] and [10].

Let also $J: B \rightarrow \mathbb{R}$ be a positive linear functional. We shall assume that $J$ is absolutely continuous with respect to $I$, i.e., for all $\varepsilon>0$ and for all $u$ in $+B$ there exists $\delta>0$ such that, for all $v$ in $+B$ with $v \leq u$ and $I(v)<\delta$ one has $J(v)<\varepsilon$ (see [1] and [3]), which is denoted by $J \ll I$.

2000 Mathematics Subject Classification: 28B05, 26D15.

This paper has been jointly written when the second author visited the Universities of Granada and Almería for scientific research, during February 1998. 
Notice that $J$ must also be a Daniell integral (due to its absolute continuity with respect to $I$ ).

According to the general theory, $I$ generates the vector lattice $L(I)$ of all $I$-integrable functions and $J$ generates the vector lattice $L(J)$ of all $J$ integrable functions. We shall denote by $L_{\mathrm{b}}(I)\left(\operatorname{resp} . L_{\mathrm{b}}(J)\right)$ the set of all bounded $I$-integrable (resp. $J$-integrable) functions. Recall that for $f: X \rightarrow$ $\mathbb{R}$, to say that $f$ is in $L(I)$ means that for every $\varepsilon>0$ there exist $g, h: X \rightarrow \mathbb{R}$ with the following three properties:

(a) There exists an increasing sequence $\left(g_{n}\right)_{n}$ in $B$ such that $g_{n} \nearrow g$ and

$$
I^{*}(g):=\sup _{n} I\left(g_{n}\right)<\infty .
$$

(b) There exists a decreasing sequence $\left(h_{n}\right)_{n}$ in $B$ such that $h_{n} \searrow h$ and

$$
I_{*}(h):=\inf _{n} I\left(h_{n}\right)>-\infty .
$$

(c) One has the inequalities $h \leq f \leq g$ and $0 \leq I^{*}(g)-I_{*}(h)<\varepsilon$ (the last inequality actually means that $\left.\sup _{n}\left[I\left(g_{n}-h_{n}\right)\right]<\varepsilon\right)$.

In case $f \geq 0$ one can suppose $h \geq 0$.

Then $I$ can be uniquely extended to a linear positive functional $\bar{I}$ : $L(I) \rightarrow \mathbb{R}$ having the property that $I_{*}(h) \leq \bar{I}(f) \leq I^{*}(g)$ for all $h$ and $g$ as above.

Similar considerations apply to the extension of $J$.

Lemma 1. If $J \ll I$, then $L_{\mathrm{b}}(I) \subset L_{\mathrm{b}}(J)$.

Proof. Let $u \geq 0$ in $L_{\mathrm{b}}(I)$. We shall prove that $u \in L(J)$ (i.e., $u \in$ $\left.L_{\mathrm{b}}(J)\right)$ and this will imply $L_{\mathrm{b}}(I) \subset L_{\mathrm{b}}(J)$, in view of the decomposition $u:=u^{+}-u^{-}$with $u^{+}, u^{-}$in $L_{\mathrm{b}}(I)$ for arbitrary $u \in L_{\mathrm{b}}(I)$.

Consider a number $M>0$ such that $u \leq M$. Take $\varepsilon>0$. Since $J \ll I$, one can find $\delta>0$ such that for all $0 \leq v \leq 2 M, v \in B$, the inequality $I(v)<\delta$ implies that $J(v)<\varepsilon$.

We can consider $h \leq u \leq g$ with $g_{n} \nearrow g, h_{n} \searrow h \geq 0, I^{*}(g)-I_{*}(h)$ $<\delta / 2$, as above. One can assume $0 \leq h_{n} \leq M, 0 \leq g_{n} \leq M$, because $g_{n} \vee 0=: g_{n}^{\prime} \nearrow g=g \vee 0$ and $g_{n}^{\prime \prime}:=g_{n}^{\prime} \wedge M \nearrow g \wedge M \geq u ; h_{n} \vee 0=: h_{n}^{\prime} \searrow h$ $=h \vee 0 ;$ and

$I^{*}(g \wedge M)-I_{*}(h)=\sup _{n}\left[I\left(g_{n}^{\prime \prime}-h_{n}^{\prime}\right)\right] \leq I^{*}(g)-I_{*}(h)=\sup _{n}\left[I\left(g_{n}-h_{n}\right)\right]<\delta / 2$.

For all $n$ one has $\left|g_{n}-h_{n}\right| \leq g_{n}+h_{n} \leq 2 M$. On the other hand, $\left|g_{n}-h_{n}\right| \rightarrow|g-h|=g-h$ pointwise. Since all $g_{n}$ and $h_{n}$ are in $L(I)$, we can use the measure space generated by $I$ and Lebesgue's Dominated Convergence Theorem to conclude that $\bar{I}(g-h)=\lim _{n} I\left(\left|g_{n}-h_{n}\right|\right)$.

Since $\bar{I}(g-h)=I^{*}(g)-I_{*}(h)<\delta / 2$, there exists a natural number $n_{0}$ such that $I\left(\left|g_{n}-h_{n}\right|\right)<\delta$ for all $n \geq n_{0}$. It follows that for all $n \geq n_{0}$ one 
has $J\left(\left|g_{n}-h_{n}\right|\right)<\varepsilon$, because $\left|g_{n}-h_{n}\right| \leq 2 M$; consequently,

$$
J\left(g_{n}-h_{n}\right) \leq J\left(\left|g_{n}-h_{n}\right|\right)<\varepsilon .
$$

Since the sequence is increasing, one gets

$$
\sup _{n} J\left(g_{n}-h_{n}\right)=J^{*}(g)-J_{*}(h) \leq \varepsilon,
$$

which means that $u \in L(I)$, because $\varepsilon$ is arbitrary.

Lemma 2. One has $\bar{J} \ll \bar{I}$ for bounded functions; i.e., for every $\varepsilon>0$ and every $M>0$, there exists $\delta>0$ having the property that if $u \in L(I)$ is such that $0 \leq u \leq M$ and $\bar{I}(u)<\delta$, then $\bar{J}(u)<\varepsilon$. Consequently, if $0 \leq u \in L(I)$ is such that $\bar{I}(u)=0$, one has $u \in L(J)$ and $\bar{J}(u)=0$.

Proof. Let $\varepsilon, M>0$. There exists $\delta_{1}>0$ such that for all $v \in B$ with $0 \leq v \leq M$ and $I(v)<\delta_{1}$ one has $J(v)<\varepsilon / 2$. Set $\delta:=\delta_{1} / 4$.

Now, take $u \in L(I)$ with $0 \leq u \leq M$ and $\bar{I}(u)<\delta$. Consider $h_{n}, g_{n}$ in $B$ with $g_{n} \nearrow g, h_{n} \searrow h, h \leq u \leq g$ and $I^{*}(g)-I_{*}(h)=\sup _{n}\left[I\left(g_{n}-h_{n}\right)\right]$ $<\delta_{1} / 4$ as above. As we have seen, one can consider that $0 \leq h_{n} \leq M$, $0 \leq g_{n} \leq M$.

Choose $n_{0} \in \mathbb{N}$ such that $\bar{J}(u) \leq J^{*}(g)=\sup _{n} J\left(g_{n}\right)<J\left(g_{n_{0}}\right)+\varepsilon / 2$; therefore

$$
\bar{J}(u)<J\left(g_{n}\right)+\varepsilon / 2, \quad \forall n \geq n_{0} .
$$

For every $n \in \mathbb{N}$, one has

$$
I\left(g_{n}\right)=I\left(g_{n}-h_{n}\right)+I\left(h_{n}\right)<I\left(h_{n}\right)+\delta_{1} / 4 .
$$

Since $I_{*}(h):=\inf _{n} I\left(h_{n}\right)$, one can find $n_{1} \in \mathbb{N}$ such that $I\left(h_{n_{1}}\right)<I_{*}(h)+$ $\delta_{1} / 4 \leq \bar{I}(u)+\delta_{1} / 4$, therefore

$$
I\left(h_{n}\right)<\bar{I}(u)+\delta_{1} / 4, \quad \forall n \geq n_{1} .
$$

Now, let $n \geq \max \left\{n_{0}, n_{1}\right\}$. In view of (2) and (3), one gets

$$
I\left(g_{n}\right)<I\left(h_{n}\right)+\delta_{1} / 4<\bar{I}(u)+\delta_{1} / 2<3 \delta_{1} / 4<\delta_{1},
$$

which implies that $J\left(g_{n}\right)<\varepsilon / 2$.

In view of (1), one has

$$
\bar{J}(u)<J\left(g_{n}\right)+\varepsilon / 2<\varepsilon
$$

and, because $\varepsilon$ is arbitrary, (4) shows that $\bar{J} \ll \bar{I}$ for bounded functions in $L(I)$.

For the case $0 \leq u \in L(I)$ with $\bar{I}(u)=0$, one has $u=\sup _{n} u_{n}$, where $\left(u_{n}\right)_{n}$ is the increasing sequence in $L_{\mathrm{b}}(I)$ given by $u_{n}:=u \wedge n$. For every $n$ one has $\bar{I}\left(u_{n}\right)=0$ and the absolute continuity for bounded functions gives $\bar{J}\left(u_{n}\right)=0$. Since $\sup _{n} \bar{J}\left(u_{n}\right)=0$, Beppo-Levi's theorem implies $u \in L(J)$, and $\bar{J}(u)=0$. 
REMARK. $\bar{J} \ll \bar{I}$ for bounded functions means that for every $\varepsilon>0$ and $0 \leq h \in L_{\mathrm{b}}(I)$, there exists $\delta>0$ having the property that if $u \in L(I)$ is such that $0 \leq u \leq h$, the inequality $\bar{I}(u)<\delta$ implies $\bar{J}(u)<\varepsilon$.

In order to continue our investigations, we introduce, for every $u \geq 0$ in $L_{\mathrm{b}}(I)$ and $\varepsilon>0$ :

(a) the average range of $\bar{J}$ with respect to $\bar{I}$ on $u$, which is the set of real numbers

$$
A(\bar{I}, \bar{J})(u):=\{\bar{J}(v) / \bar{I}(v) ; 0 \leq v \leq u, v \in L(I), \bar{I}(v)>0\}
$$

(b) the $\varepsilon$-approximate average range of $\bar{J}$ with respect to $\bar{I}$ on $u$, which is the set of real numbers (possibly empty)

$$
A_{\varepsilon}(\bar{I}, \bar{J})(u):=\{x \in \mathbb{R} ;|x-a| \leq \varepsilon \text { for all } a \in A(\bar{I}, \bar{J})(u)\} .
$$

We make three assumptions which will be discussed and justified at the end of the paper. $\mathbb{N}$ denotes the set $\{1,2,3, \ldots\}$ of all natural numbers.

Assumption 1. This assumption is sequential and inductive, consisting of the following sequence of steps:

$s(1)$ : There exists a sequence $\left(h_{n ; 1}\right)_{n \in \mathbb{N}}$ or a finite family $\left(h_{n ; 1}\right)_{1 \leq n \leq p_{1}}$ of positive functions in $L(I)$ such that $\bar{I}\left(h_{n ; 1}\right)>0$ for all $n$ and

$$
\sum_{n} h_{n ; 1}=1
$$

with pointwise covergence.

$s(2)$ : For every $n \in \mathbb{N}$ or $1 \leq n \leq p_{1}$, there exists a sequence $\left(h_{(n, i) ; 2}\right)_{i \in \mathbb{N}}$ or a finite family $\left(h_{(n, i) ; 2}\right)_{1 \leq i \leq p_{2}}$ of positive functions in $L(I)$ such that $\bar{I}\left(h_{\alpha ; 2}\right)>0$ for all possible $\alpha:=(n, i)$, and for all possible $n$ we have pointwise

$$
\sum_{i} h_{(n, i) ; 2}=h_{n ; 1} \text {. }
$$

This implies $\sum_{\alpha} h_{\alpha ; 2}=1$, where the sum $\sum_{\alpha}$ is taken pointwise over the set of all possible $\alpha$.

Assuming that the step $s(n-1)$ for $n \geq 2$ of the assumption has been defined (this pertains to the family $\left(h_{\alpha ; n-1}\right)_{\alpha}$ where $\alpha \in \mathbb{N}^{n-1}$ ranges over all possible $\alpha$ ) we shall write $\left(\alpha, i_{n}\right) \in \mathbb{N}^{n}$, for every $\alpha=\left(i_{1}, \ldots, i_{n-1}\right) \in \mathbb{N}^{n-1}$ and $i_{n} \in \mathbb{N}$.

Now we are able to write the next step:

$s(n)$ : For every $\alpha \in \mathbb{N}^{n-1}$ in the set of all possible $\alpha$ given by the previous steps, there exists a sequence $\left(h_{(\alpha, i) ; n}\right)_{i \in \mathbb{N}}$ or a finite set $\left(h_{(\alpha, i) ; n}\right)_{1 \leq i \leq p_{n}}$ of positive functions in $L(I)$ such that $\bar{I}\left(h_{\beta ; n}\right)>0$ for all possible $\beta$. Moreover, 
for all possible $\alpha$, we have pointwise

(i) $n$

$$
\sum_{i} h_{(\alpha, i) ; n}=h_{\alpha ; n-1}
$$

where the sum $\sum_{i}$ is taken over the set of all possible $i$. This implies, in view of $\sum_{\alpha} h_{\alpha ; n-1}=1$ in $s(n-1)$ and in view of $(\mathrm{i})_{n}$, that $\sum_{\beta} h_{\beta ; n}=1$, where $\beta$ ranges over the set of all possible $\beta$.

Final comment upon Assumption 1: For every possible $\alpha \in \mathbb{N}^{m}$, if $n>m$, one has

$$
h_{\alpha ; m}=\sum_{\beta} h_{(\alpha, \beta) ; n}
$$

where the sum runs over all possible $\beta \in \mathbb{N}^{n-m}$, with obvious notations. Note that all the $h_{\alpha ; m}$ are in $L_{\mathrm{b}}(I)$.

Assumption 2. For every natural number $n$ and for every $\alpha \in \mathbb{N}^{n}$ in the set of all possible $\alpha$, one has

$$
A_{2^{-n}}(\bar{I}, \bar{J})\left(h_{\alpha ; n}\right) \neq \emptyset .
$$

Assumption 3. There exists a number $M>0$ such that for all $n$ in $\mathbb{N}$ and for all $\alpha \in \mathbb{N}^{n}$ in the set of all possible $\alpha$, one has

$$
A_{2^{-n}}(\bar{I}, \bar{J})\left(h_{\alpha ; n}\right) \subset[-M, M] .
$$

The general theory says that if $f$ is a bounded function in $L(I)$ and $u$ is in $L(I)$, then $f u$ is in $L(I)$. We can now state the main result of this paper.

Theorem (An exact Radon-Nikodym theorem for Daniell integrals). Assume that $I, J$ are as above and Assumptions 1-3 are fulfilled. Then there exists a positive bounded function $f$ in $L(I)$ such that

$$
\bar{J}(u)=\bar{I}(f u)
$$

for all $u$ in $L(I)$. The function $f$ (called the Radon-Nikodym derivative of $\bar{J}$ with respect to $\bar{I})$ is I-almost unique, which means that if $g$ in $L(I)$ is such that $\bar{J}(u)=\bar{I}(g u)$ for all $u$ in $L(I)$ then $\bar{I}(|f-g|)=0$.

Proof. We shall construct a sequence $\left(f_{n}\right)_{n}$ of bounded $I$-integrable functions.

Let $n$ be in $\mathbb{N}$. In order to construct $f_{n}$, we take an element $r_{\alpha ; n}$ in each $A_{2^{-n}}(\bar{I}, \bar{J})\left(h_{\alpha ; n}\right)$ for all possible $\alpha$ in $\mathbb{N}^{n}$, according to Assumption 2. We define $f_{n}: X \rightarrow \mathbb{R}$ pointwise by

$$
f_{n}:=\sum_{\alpha} r_{\alpha ; n} h_{\alpha ; n}
$$

(where $\alpha \in \mathbb{N}^{n}$ ranges over the set of all possible $\alpha$ ). 
One has clearly

$$
\left|f_{n}\right| \leq \sum_{\alpha}\left|r_{\alpha ; n}\right| h_{\alpha ; n} \leq M \sum_{\alpha} h_{\alpha ; n}=M,
$$

so $f_{n}$ is bounded. Here we have used Assumption 3 and again Assumption 1. If $\mu$ is the measure induced by the Daniell integral $I$ (according to the general theory), then the functions $f_{n}$ are clearly $\mu$-measurable and, being bounded, are also $\mu$-integrable, i.e., they are in $L(I)$.

Now we prove that the sequence $\left(f_{n}\right)$ is uniformly Cauchy, which implies that it is uniformly convergent to a function $f$. Indeed, let $m<n$ in $\mathbb{N}$. We shall prove that for all $t$ in $X$ one has

$$
\left|f_{m}(t)-f_{n}(t)\right| \leq 2^{1-m}
$$

and this will prove the assertion.

Take $t \in X$. We have (here $\beta \in \mathbb{N}^{n-m}$ is taken to be in the set of all possible such indices)

$$
\begin{aligned}
\left|f_{m}(t)-f_{n}(t)\right| & =\left|\sum_{\alpha} r_{\alpha ; m} h_{\alpha ; m}(t)-\sum_{\gamma} r_{\gamma ; n} h_{\gamma ; n}(t)\right| \\
& \leq \sum_{\alpha}\left|r_{\alpha ; m} h_{\alpha ; m}(t)-\sum_{\beta} r_{(\alpha, \beta) ; n} h_{(\alpha, \beta) ; n}(t)\right| \\
& \leq \sum_{(\alpha, \beta)}\left|r_{\alpha ; m} h_{(\alpha, \beta) ; n}(t)-r_{(\alpha, \beta) ; n} h_{(\alpha, \beta) ; n}(t)\right| \\
& =\sum_{(\alpha, \beta)} h_{(\alpha, \beta) ; n}(t)\left|r_{\alpha ; m}-r_{(\alpha, \beta) ; n}\right|
\end{aligned}
$$

(see the final comment upon Assumption 1).

For every $(\alpha, \beta)$ we take a natural $i$ such that (with obvious notation)

$$
0 \leq h_{(\alpha, \beta, i) ; n+1}=: v \leq h_{(\alpha, \beta) ; n} \leq h_{\alpha ; m}, \quad \bar{I}(v)>0 .
$$

Summing upon all possible $(\alpha, \beta)$ and finding each time such a $v=$ $v(\alpha, \beta)$, one has

$$
\begin{aligned}
\left|f_{m}(t)-f_{n}(t)\right| & \leq \sum_{(\alpha, \beta)} h_{(\alpha, \beta) ; n}(t)\left(\left|r_{\alpha ; m}-\frac{\bar{J}(v)}{\bar{I}(v)}\right|+\left|\frac{\bar{J}(v)}{\bar{I}(v)}-r_{(\alpha, \beta) ; n}\right|\right) \\
& \leq \sum_{(\alpha, \beta)} h_{(\alpha, \beta) ; n}(t)\left(2^{-m}+2^{-n}\right) \\
& \leq 2^{-m+1} \sum_{(\alpha, \beta)} h_{(\alpha, \beta) ; n}(t)=2^{-m+1}
\end{aligned}
$$

and (5) is proved.

Let $\tilde{f}: X \rightarrow \mathbb{R}$ be the (uniform) limit $\tilde{f}:=\lim _{n} f_{n}$. 
It is clear (because $\left|f_{n}\right| \leq M$ ) that $|\widetilde{f}| \leq M$ and so $\widetilde{f}$ is bounded, therefore $\tilde{f} \in L(I)$ according to the general theory.

We prove that for all $u$ in $L(I)$,

$$
\bar{J}(u)=\bar{I}(\widetilde{f} u) .
$$

We show that (6) holds for every positive bounded $u \leq 1$ in $L(I)$. Indeed, one can write $\lim _{n} f_{n} u=\widetilde{f} u$ and $\left|f_{n} u\right| \leq M,|\widetilde{f} u| \leq M$ (everything pointwise) and this implies

$$
\lim \bar{I}\left(f_{n} u\right)=\bar{I}(\widetilde{f} u) .
$$

On the other hand, for every $n$ in $\mathbb{N}$,

$$
\left|\bar{J}(u)-\bar{I}\left(f_{n} u\right)\right|=\left|\bar{J}(u)-\bar{I}\left(\left(\sum_{\alpha} r_{\alpha ; n} h_{\alpha ; n}\right) u\right)\right|=\left|\bar{J}(u)-\sum_{\alpha} r_{\alpha ; n} \bar{I}\left(u h_{\alpha ; n}\right)\right|
$$

(again by dominated convergence).

Because $u=u \sum_{\alpha} h_{\alpha ; n}$, one also has $\bar{J}(u)=\sum_{\alpha} \bar{J}\left(u h_{\alpha ; n}\right)$ and so

$$
\left|\bar{J}(u)-\bar{I}\left(f_{n} u\right)\right|=\left|\sum_{\alpha}\left(\bar{J}\left(u h_{\alpha ; n}\right)-r_{\alpha ; n} \bar{I}\left(u h_{\alpha ; n}\right)\right)\right| .
$$

In case $\bar{I}\left(u h_{\alpha ; n}\right)=0$ one has $\bar{J}\left(u h_{\alpha ; n}\right)=0$, because $\bar{J} \ll \bar{I}$. In case $\bar{I}\left(u h_{\alpha ; n}\right)>0$ one has $0 \leq u h_{\alpha ; n} \leq h_{\alpha ; n}$ and then

$$
\left|\frac{\bar{J}\left(u h_{\alpha ; n}\right)}{\bar{I}\left(u h_{\alpha ; n}\right)}-r_{\alpha ; n}\right| \leq 2^{-n},
$$

which implies in all situations that

$$
\left|\bar{J}\left(u h_{\alpha ; n}\right)-r_{\alpha ; n} \bar{I}\left(u h_{\alpha ; n}\right)\right| \leq 2^{-n} \bar{I}\left(u h_{\alpha ; n}\right) \leq 2^{-n} \bar{I}\left(h_{\alpha ; n}\right) .
$$

In view of (8) and (9), one obtains

$$
\left|\bar{J}(u)-\bar{I}\left(f_{n} u\right)\right| \leq 2^{-n} \sum_{\alpha} \bar{I}\left(h_{\alpha ; n}\right)=2^{-n} \bar{I}(1),
$$

which implies

$$
\lim \bar{I}\left(f_{n} u\right)=\bar{J}(u) .
$$

From (7) and (10) we obtain (6), which therefore holds for positive bounded functions $u$ in $L(I)$.

If $u$ is an arbitrary positive function in $L(I)$, we have the pointwise convergence $u_{n} \nearrow u$, where $u_{n}:=u \wedge n$. Since $\bar{J}\left(u_{n}\right)=\bar{I}\left(\widetilde{f} u_{n}\right)$ for all $n$, it follows, by passing to suprema, that $\bar{J}(u)=\bar{I}(\widetilde{f} u)$ and (6) is true for all positive functions in $L(I)$. By linearity, (6) holds for all functions in $L(I)$. 
If $\mu$ is the (complete) measure induced by the Daniell integral $I$, then (6) implies $\left(\chi_{A}=\right.$ the indicator function of $\left.A\right)$

$$
0 \leq \bar{J}\left(\chi_{A}\right)=\int_{A} \tilde{f} d \mu
$$

for all $A \subset X$ with $\chi_{A} \in L(I)$. General measure theory says that $\widetilde{f}(t) \geq 0$ $\mu$-almost everywhere.

The set $M:=\{t \in X ; \widetilde{f}(t)<0\}$ has the properties $\chi_{M} \in L(I)$ and $\mu(M)=\bar{I}\left(\chi_{M}\right)=0$. Defining $f: X \rightarrow \mathbb{R}$ via

$$
f(t):= \begin{cases}\widetilde{f}(t), & t \notin M \\ 0, & t \in M\end{cases}
$$

one has $f \geq 0$ everywhere, $f=\tilde{f} \mu$-almost everywhere and therefore

$$
\bar{J}(u)=\bar{I}(\widetilde{f} u)=\bar{I}(f u)
$$

for all $u$ in $L(I)$.

For the unicity, consider another function $g$ in $L(I)$ such that $\bar{J}(u)=$ $\bar{I}(g u)$ for all $u$ in $L(I)$. So, we have $\bar{I}\left(f \chi_{A}\right)=\bar{I}\left(g \chi_{A}\right)$, which means that $\int_{A} f d \mu=\int_{A} g d \mu$ for all $A \subset X$ with $\chi_{A} \in L(I)$. General measure theory says that $g=f \mu$-almost everywhere, which means

$$
0=\int|f-g| d \mu=\bar{I}(|f-g|) .
$$

\section{Other results and comments}

3.1. We begin with a general result which will furnish material for some comments. Assume therefore that $X \neq \emptyset$ is an abstract set, $B$ a vector lattice of functions $f: X \rightarrow \mathbb{R}$ and $I, J: B \rightarrow \mathbb{R}$ are linear positive functionals. Using the conventions $\frac{0}{0}:=0$ and $\frac{a}{0}:=\infty$ for $a>0$ we shall modify the previous definitions a little. Namely, for every $u$ in $+B$ and $\varepsilon>0$, we set

$$
\begin{aligned}
& A^{\prime}(I, J)(u):=\{J(v) / I(v) ; 0 \leq v \leq u, v \in B\}, \\
& A_{\varepsilon}^{\prime}(I, J)(u):=\left\{x \in \mathbb{R} ;|x-a| \leq \varepsilon, a \in A^{\prime}(I, J)(u)\right\} .
\end{aligned}
$$

Proposition. (i) Assume that for all $u$ in $+B$, the set $A^{\prime}(I, J)(u)$ is bounded (e.g. in case there exists a number $M>0$ such that $J \leq M I)$. Then $J \ll I$.

(ii) For every $u$ in $+B$ and every $\varepsilon>0$, the set $A_{\varepsilon}^{\prime}(I, J)(u)$ is closed (actually compact).

(iii) For every $u$ in $+B$ we have $0<\varepsilon<\gamma \Rightarrow A_{\varepsilon}^{\prime}(I, J)(u) \subset A_{\gamma}^{\prime}(I, J)(u)$.

(iv) Assuming that $u$ in $+B$ is such that $A_{\varepsilon}^{\prime}(I, J)(u) \neq \emptyset$ for all $\varepsilon>0$, the intersection $\bigcap_{\varepsilon>0} A_{\varepsilon}^{\prime}(I, J)(u)$ contains exactly one point.

Proof. (i) Assume that for all $u$ in $+B$ the set $A^{\prime}(I, J)(u)$ is bounded. If $J \ll I$ is false, we can find $\varepsilon_{0}>0$ and $u$ in $+B$ with the property that 
for all $n$ in $\mathbb{N}$, there exists $0 \leq u_{n} \leq u$ in $B$ such that $I\left(u_{n}\right)<1 / n$ and $J\left(u_{n}\right) \geq \varepsilon_{0}$.

If $I\left(u_{n}\right)=0$, then $J\left(u_{n}\right) / I\left(u_{n}\right)=\infty$ and $A^{\prime}(I, J)(u)$ is not bounded.

If $I\left(u_{n}\right)>0$, then $J\left(u_{n}\right) / I\left(u_{n}\right) \geq n \varepsilon_{0}$ and, in this case too, $A^{\prime}(I, J)(u)$ is not bounded. Contradiction, and (i) follows.

In the particular case when $J \leq M I$ for some positive $M$, one has obviously $A^{\prime}(I, J)(u) \subset[0, M]$.

Assertion (ii) is clear when $A_{\varepsilon}^{\prime}(I, J)(u)=\emptyset$. So, assume $A_{\varepsilon}^{\prime}(I, J)(u) \neq \emptyset$.

If a number $x$ is such that $x=\lim _{n} x_{n}$, where $\left(x_{n}\right)_{n}$ is a sequence in $A_{\varepsilon}^{\prime}(I, J)(u)$, then for an arbitrary fixed $0 \leq v \leq u$ in $B$ one has

$$
\left|\frac{J(v)}{I(v)}-x_{n}\right| \leq \varepsilon
$$

for every $n$. Passing to the limit gives

$$
\left|\frac{J(v)}{I(v)}-x\right| \leq \varepsilon
$$

The fact that $v$ is arbitrary shows that $x \in A_{\varepsilon}^{\prime}(I, J)(u)$.

Point (iii) is trivial. We prove (iv).

For every $\varepsilon>0$, the nonempty set $A_{\varepsilon}^{\prime}(I, J)(u)$ is bounded (for every $x$ and $y$ in $A_{\varepsilon}^{\prime}(I, J)(u)$ one has $|x-y| \leq|x-a|+|y-a| \leq 2 \varepsilon$, upon taking some $a$ in $A_{\varepsilon}^{\prime}(I, J)(u)$ ), therefore compact, and the decreasing intersection is nonempty. Put $A:=\bigcap_{\varepsilon>0} A_{\varepsilon}^{\prime}(I, J)(u)$.

Assume the existence of $x \neq y$ in $A$; then one has, for a fixed $0 \leq v \leq u$ in $B$, the inequalities

Hence

$$
\left|\frac{J(v)}{I(v)}-x\right| \leq \frac{|x-y|}{4} \text { and }\left|\frac{J(v)}{I(v)}-y\right| \leq \frac{|x-y|}{4} .
$$

which is false.

$$
|x-y| \leq\left|\frac{J(v)}{I(v)}-x\right|+\left|\frac{J(v)}{I(v)}-y\right| \leq \frac{|x-y|}{2},
$$

Commenting on the Proposition, we can say:

(a) Point (i) motivates Assumption 3 a little. For example, in the particular case when there exists a positive number $M$ such that $J \leq M I$, one quickly sees that $\bar{J} \leq M \bar{I}$ and this implies that for every positive $u$ in $L(I)$ one has $A^{\prime}(I, J)(u) \subset[0, M]$.

(b) Point (iv) can give us some ideas in connection with the possible values of the function $f$. Namely, they should be close to the elements in the intersection of the form

$$
\bigcap_{\varepsilon>0} A_{\varepsilon}^{\prime}(\bar{I}, \bar{J})\left(h_{\alpha ; n}\right)
$$

for large $n$. So, $f$ is obtained via a kind of differentiation procedure. 
(c) In connection with Assumption 2, which says that for large $n$ the average range $A(\bar{I}, \bar{J})\left(h_{\alpha ; n}\right)$ must have very small diameter (see also comment (b)), the following example will be, perhaps, illuminating, putting into evidence a concrete construction of the family $\left(h_{\alpha ; n}\right)$.

3.2. We now give an example to show how the theorem effectively works.

We take $X:=[0,1]$ and $B:=$ the algebra of all continuous functions $u:[0,1] \rightarrow \mathbb{R}$. The functional $I: B \rightarrow \mathbb{R}$ is given by $I(f):=\int_{0}^{1} u(x) d x$. In order to construct $J$ we fix a positive function $f \in B$ and then $J: B \rightarrow \mathbb{R}$ is given by $J(u):=\int_{0}^{1} f(x) u(x) d x$.

If $M:=\max \{f(x) ; x \in[0,1]\}$ then $J \leq M I$, which shows that $J \ll I$ and Assumption 3 is automatically satisfied.

One knows that $\bar{I}$ is exactly the Lebesgue integral on the space $L(I)$ of all Lebesgue integrable functions, so

$$
\bar{I}(u)=\int u d \mu, \quad \forall u \in L(I),
$$

where $\mu: \mathcal{M} \rightarrow+\mathbb{R}$ is the Lebesgue measure (induced by $I$ over the set $\mathcal{M}$ of all Lebesgue measurable subsets of $[0,1])$. Then $\bar{J}$ acts via

$$
\bar{J}(u)=\int f u d \mu, \quad \forall u \in L(I) .
$$

We now show how Assumptions 1 and 2 can be satisfied. To this end, we use the following

StATEMENT. Let $U \subset \mathbb{R}$ be a compact interval, $\mu$ the Lebesgue measure on $U$ and $f: U \rightarrow \mathbb{R}$ a positive continuous function. Then, for every $\varepsilon>0$, there exists $\delta>0$ such that for every $a$ in $U$ which is not the right end of $U$, one has the property: for each interval $[a, b] \subset U$ with $b-a<\delta$ and for each Lebesgue integrable function $u: U \rightarrow \mathbb{R}$ such that $0 \leq u \leq \chi_{[a, b]}$ and $u$ is not null $\mu$-almost everywhere on $[a, b]$, the following relation holds:

$$
\frac{\int_{[a, b]} f u d \mu}{\int_{[a, b]} u d \mu} \in[f(a)-\varepsilon / 2, f(a)+\varepsilon / 2] .
$$

Proof. Since $f$ is bounded, there exists a natural number $k$, not depending on $a$, such that

$$
\frac{1}{k}+\frac{1}{k^{2}}+\frac{f(a)}{k}<\frac{\varepsilon}{2} .
$$

In view of the uniform continuity of $f$, there exists $\delta>0$ not depending on $a$ such that

$$
M:=\sup \{f(x) ; x \in[a, b]\} \leq f(a)+1 / k
$$

and

$$
m:=\inf \{f(x) ; x \in[a, b]\} \geq f(a)-1 / k
$$

if $[a, b] \subset U$ is such that $b-a<\delta$. 
Fix the interval $[a, b]$ as in the Statement. First, we shall prove it for every Riemann integrable function

$$
u \in H(a, b):=\left\{u: U \rightarrow \mathbb{R} ; 0 \leq u \leq \chi_{[a, b]}, u \neq 0 \mu \text {-a.e. }\right\} .
$$

There exist positive continuous functions $g, h:[a, b] \rightarrow \mathbb{R}$ such that

$$
0 \leq h \leq u \leq g \text { and } \int_{[a, b]} h d \mu>0
$$

(since $\int_{[a, b]} u d \mu>0$ ).

In view of the general properties of the Daniell integral, we can find an increasing sequence $\left(h_{n}\right)_{n}$ of positive continuous functions $h_{n}:[a, b] \rightarrow \mathbb{R}$, $h_{n} \leq u$, and a decreasing sequence $\left(g_{n}\right)_{n}$ of positive continuous functions $g_{n}:[a, b] \rightarrow \mathbb{R}, g_{n} \geq u$, such that

$$
\int_{[a, b]} u d \mu=\sup _{n} \int_{[a, b]} h_{n} d \mu=\inf _{n} \int_{[a, b]} g_{n} d \mu .
$$

One can find a natural $n$ such that

$$
1 \leq \frac{\int_{[a, b]} g_{n} d \mu}{\int_{[a, b]} h_{n} d \mu} \leq 1+\frac{1}{k} \quad \text { and } \quad 1 \geq \frac{\int_{[a, b]} h_{n} d \mu}{\int_{[a, b]} g_{n} d \mu} \geq 1-\frac{1}{k} .
$$

Put $h:=h_{n}$ and $g:=g_{n}$. One has

$$
\frac{\int_{[a, b]} f h d \mu}{\int_{[a, b]} g d \mu} \leq \frac{\int_{[a, b]} f u d \mu}{\int_{[a, b]} u d \mu} \leq \frac{\int_{[a, b]} f g d \mu}{\int_{[a, b]} h d \mu} .
$$

But using (12)-(14) gives

$$
\int_{[a, b]} f g d \mu \leq M \int_{[a, b]} g d \mu \leq\left(f(a)+\frac{1}{k}\right) \int_{[a, b]} g d \mu
$$

therefore,

$$
\frac{\int_{[a, b]} f g d \mu}{\int_{[a, b]} h d \mu} \leq\left(f(a)+\frac{1}{k}\right) \frac{\int_{[a, b]} g d \mu}{\int_{[a, b]} h d \mu} \leq\left(f(a)+\frac{1}{k}\right)\left(1+\frac{1}{k}\right)
$$

and

$$
\int_{[a, b]} f h d \mu \geq m \int_{[a, b]} h d \mu \geq\left(f(a)-\frac{1}{k}\right) \int_{[a, b]} h d \mu
$$

therefore

$$
\frac{\int_{[a, b]} f h d \mu}{\int_{[a, b]} g d \mu} \geq\left(f(a)-\frac{1}{k}\right) \frac{\int_{[a, b]} h d \mu}{\int_{[a, b]} g d \mu} \geq\left(f(a)-\frac{1}{k}\right)\left(1-\frac{1}{k}\right) .
$$


It follows that

$$
f(a)-\left(\frac{1}{k}+\frac{f(a)}{k}-\frac{1}{k^{2}}\right) \leq \frac{\int_{[a, b]} f u d \mu}{\int_{[a, b]} u d \mu} \leq f(a)+\left(\frac{1}{k}+\frac{f(a)}{k}+\frac{1}{k^{2}}\right),
$$

which implies, using (11), that

$$
f(a)-\frac{\varepsilon}{2} \leq \frac{\int_{[a, b]} f u d \mu}{\int_{[a, b]} u d \mu} \leq f(a)+\frac{\varepsilon}{2} .
$$

Now, we prove the validity of the result for every Lebesgue integrable function $u \in H(a, b)$. There exists a sequence $\left(u_{n}\right)_{n}$ of Riemann integrable functions, $u_{n}: U \rightarrow \mathbb{R}$, such that $\left\|u_{n}-u\right\|_{1}:=\int\left|u_{n}-u\right| d \mu \rightarrow 0$ and one can suppose that $u_{n} \rightarrow u \mu$-a.e. Then $\left\|u_{n}^{+}-u\right\|_{1} \rightarrow 0$, where $u_{n}^{+}:=u_{n} \vee 0$ (since $u=u \vee 0$ ) and hence $\left\|z_{n}-u\right\|_{1} \rightarrow 0$, where $z_{n}:=u_{n}^{+} \wedge \chi_{[a, b]}$ (since $\left.u \wedge \chi_{[a, b]}=u\right)$. We have used the properties of the Banach lattice $L^{1}(\mu)$. One sees that $0 \leq z_{n} \leq \chi_{[a, b]}, z_{n}$ are Riemann integrable and, for $n$ greater than some $n_{0}$, one must have $\int z_{n} d \mu>0$, because $\int u d \mu>0$.

The result already obtained for Riemann integrable functions yields for all $n \geq n_{0}$,

$$
\frac{\int_{[a, b]} f z_{n} d \mu}{\int_{[a, b]} z_{n} d \mu} \in[f(a)-\varepsilon / 2, f(a)+\varepsilon / 2],
$$

and passing to the $n$-limit, one obtains

$$
\frac{\int_{[a, b]} f u d \mu}{\int_{[a, b]} u d \mu} \in[f(a)-\varepsilon / 2, f(a)+\varepsilon / 2] .
$$

Using this Statement, one can see that, for given $\varepsilon>0$, there exists $\delta>0$ such that for every $0 \leq a<b \leq 1$ with $b-a<\delta$, one has

$$
A_{\varepsilon}(\bar{I}, \bar{J})\left(\chi_{[a, b]}\right) \neq \emptyset \text {. }
$$

Indeed, we saw that for given $\varepsilon>0$, one can find $\delta>0$ such that for every $a, b$ as above one has

$$
A(\bar{I}, \bar{J})\left(\chi_{[a, b]}\right) \subset[f(a)-\varepsilon / 2, f(a)+\varepsilon / 2] .
$$

Then it is immediately seen that

$$
[f(a)-\varepsilon / 2, f(a)+\varepsilon / 2] \subset A_{\varepsilon}(\bar{I}, \bar{J})\left(\chi_{[a, b]}\right) .
$$

We are prepared to satisfy Assumptions 1 and 2. The constructions indicated in the successive steps $s(1), s(2), \ldots$ will give each time a finite number of results. More precisely:

$s(1)$ : There exists a natural number $p_{1}$ such that for every interval $[a, b]$ $\subset[0,1]$ with $b-a \leq 1 / p_{1}$, one has

as we have seen.

$$
A_{2^{-1}}(\bar{I}, \bar{J})\left(\chi_{[a, b]}\right) \neq \emptyset
$$


Let us divide $[0,1]$ as follows:

$$
0=: x_{0}<x_{1}<\ldots<x_{p_{1}}:=1, \quad x_{i}-x_{i-1}=1 / p_{1} .
$$

Then we define $\left(h_{i ; 1}\right)_{1 \leq i \leq p_{1}}$ via

$$
h_{i ; 1}:= \begin{cases}\chi_{\left[x_{i-1}, x_{i}[\right.} \in L(I) & \text { for } i<p_{1}, \\ \chi_{\left[x_{i-1}, x_{i}\right]} \in L(I) & \text { for } i=p_{1} .\end{cases}
$$

$s(2)$ : There exists a natural number $p_{2}$ such that for every interval $[a, b]$ $\subset[0,1]$ with $b-a \leq 1 / p_{2}$, one has

$$
A_{2^{-2}}(\bar{I}, \bar{J})\left(\chi_{[a, b]}\right) \neq \emptyset .
$$

Then, for every $i=1, \ldots, p_{1}$, we divide $\left[x_{i-1}, x_{i}\right]$ as follows:

$$
x_{i-1}=: x_{i, 0}<x_{i, 1}<\ldots<x_{i, p_{2}}:=x_{i}, \quad x_{i, k}-x_{i, k-1}=\frac{1}{p_{1} p_{2}} .
$$

Fixing $i=1, \ldots, p_{1}-1$, we can define the functions $\left(h_{(i, k) ; 2}\right)_{1 \leq k \leq p_{2}}$ by

$$
h_{(i, k) ; 2}:=\chi_{\left[x_{i, k-1}, x_{i, k}[\right.} \in L(I)
$$

and for $i=p_{1}$ we define $\left(h_{(i, k) ; 2}\right)_{1 \leq k \leq p_{2}}$ by

$$
h_{(i, k) ; 2}:= \begin{cases}\chi_{\left[x_{i, k-1}, x_{i, k}\right.} \in L(I) & \text { for } k<p_{2}, \\ \chi_{\left[x_{i, k-1}, x_{i, k}\right]} \in L(I) & \text { for } k=p_{2} .\end{cases}
$$

We obtained the set of functions $\left(h_{\alpha ; 2}\right)_{\alpha \in A}$ with $A:=\left\{1, \ldots, p_{1}\right\} \times\left\{1, \ldots, p_{2}\right\}$ and this accomplishes the construction for step $s(2)$.

The procedure continues in the same manner (dividing all intervals into small subintervals of equal length a.s.o.).

The reader can see that, in this way, Assumptions 1 and 2 are satisfied.

One should add that the construction will give as uniform limit of the sequence $\left(f_{n}\right)_{n}$ so obtained a function which must be $\mu$-almost everywhere equal to the initial $f$, which is the Radon-Nikodym derivative of $J$ with respect to $I$.

3.3. We end with some supplementary considerations.

Our theorem gives an exact Radon-Nikodym derivative. One knows (see [1]) that for Daniell integrals one cannot generally find exact RadonNikodym derivatives, only approximate Radon-Nikodym derivatives existing always. The price payed in order to obtain this better situation was the following:

(a) We are obliged to work for the Daniell case, more particular than the case of general linear positive functionals. This general extension procedure has been studied in [2], being among the first ones concerned with Loomis systems.

(b) Additional assumptions 1-3 were adopted. 
(c) The exact Radon-Nikodym derivative one can find generally belongs to the space $L(I)$, which is considerably larger than the initial space $B$.

The procedure presented is constructive, which distinguishes the present work from [1].

\section{References}

[1] E. de Amo, I. Chitescu and M. Díaz Carrillo, An approximate functional RadonNikodym theorem, Rend. Circ. Mat. Palermo 48 (1999), 443-450.

[2] P. Bobillo Guerrero and M. Díaz Carrillo, Summable and integrable functions with respect to any Loomis system, Arch. Math. (Basel) 49 (1987), 245-256.

[3] N. Bourbaki, Intégration (Les structures fondamentales de l'analyse, livre VI), Hermann, Paris, 1956.

[4] R. Cristescu, Ordered Vector Spaces and Linear Operators, Ed. Academiei-Abacus Press, 1976.

[5] N. Dinculeanu, Vector Measures, Pergamon Press, New York, 1967.

[6] J. W. Hagood, A Radon-Nikodym theorem and $L_{p}$ completeness for finitely additive vector measures, J. Math. Anal. Appl. 113 (1968), 266-279.

[7] P. R. Halmos, Measure Theory, Van Nostrand, Princeton, 1950.

[8] H. B. Maynard, A Radon-Nikodym theorem for finitely additive bounded vector measures, Pacific J. Math. 83 (1979), 401-413.

[9] W. F. Pfeffer, Integrals and Measures, Dekker, New York, 1977.

[10] A. C. Zaanen, Integration, North-Holland, Amsterdam, 1967.

Departamento de Álgebra y Análisis Matemático

Universidad de Almería

04120 Almería, Spain

E-mail: edeamo@ualm.es

Departamento de Análisis Matemático

Universidad de Granada

18071 Granada, Spain

E-mail: madiaz@ugr.es
Faculty of Mathematics University of Bucharest

Str. Academiei, 14

Bucureşti, Romania 